\title{
In-line Mach-Zehnder interferometer with D-shaped fiber grating for temperature- discriminated directional curvature measurement
}

\author{
Biqiang Jiang, Zhiyong Bai, Changle Wang, Yunhe Zhao, Jianlin Zhao, Lin Zhang, and Kaiming Zhou
}

\begin{abstract}
A high-sensitivity curvature sensing configuration is implemented by using a fiber Mach-Zehnder interferometer (MZI) with D-shaped fiber Bragg grating (FBG). A segment of D-shaped fiber is fusion spliced into a single mode fiber at both sides, and then a short FBG is inscribed in the D-shaped fiber. The fiber device yields a significant spectrum sensitivity as high as 87.7 $\mathrm{nm} / \mathrm{m}^{-1}$ to the ultra-low curvature range from 0 to $0.3 \mathrm{~m}^{-1}$, and can distinguish the orientation of curvature experienced by the fiber as the attenuation dip producing either a blue or red wavelength shift, by virtue of the asymmetry of D-shaped fiber cladding. In addition, by tracking both resonant wavelengths of the MZI and embedded FBG, the temperature and curvature can be measured simultaneously.
\end{abstract}

Index Terms-Mach-Zehnder interferometer (MZI), D-shaped fiber, fiber Bragg grating (FBG), curvature measurement.

\section{INTRODUCTION}

$\mathrm{T}$ HE measurement of curvature plays an important role in various applications including composite material structures, astronautics, industrial robots, structural health monitoring, etc. Due to their merits of high sensitivity, small size, immunity to electromagnetic interference, optical fiber-based curvature sensors have been attracting increasing research interest. A variety of optical fiber structures have been developed for curvature or bending measurement, including the

This work was supported in part by the National Natural Science Foundation of China under Grants 61505165 and 61775182 , in part by the Natural Science Basic Research Plan in Shaanxi Province of China under Grant 2016JQ6032, and in part by Marie Skłodowska-Curie Individual Fellowships in the European Union's Horizon 2020 Research and Innovation Programme under Grant 660648.

B. Jiang is with the MOE Key Laboratory of Material Physics and Chemistry under Extraordinary Conditions and Shaanxi Key Laboratory of Optical Information Technology, School of Science, Northwestern Polytechnical University, Xi'an 710072, China, and also with the Aston Institute of Photonic Technologies, Aston University, Birmingham B4 7ET, UK (email: bqjiang@nwpu.edu.cn).

Z. Bai, C. Wang, Y. Zhao, and L. Zhang are with the Aston Institute of Photonic Technologies, Aston University, Birmingham B4 7ET, UK. (email: z.bai@hotmail.com; wangc15@aston.ac.uk; yhz@shu.edu.cn; 1.zhang@aston.ac.uk)

J. Zhao is with the MOE Key Laboratory of Material Physics and Chemistry under Extraordinary Conditions and Shaanxi Key Laboratory of Optical Information Technology, School of Science, Northwestern Polytechnical University, Xi'an 710072, China. (email: jlzhao@nwpu.edu.cn)

K. Zhou is with the Aston Institute of Photonic Technologies, Aston University, Birmingham B4 7ET, UK, and also with the State Key Laboratory of Transient Optics and Photonics, Xi'an Institute of Optics and Precision Mechanics, Chinese Academy of Sciences, Xi'an 710119, China. (email: k.zhou@aston.ac.uk) fiber grating-based [1-9], interferometer-based schemes [10-18], etc. For fiber grating-based structures, such as fiber Bragg gratings (FBGs) written in multi-core fiber (MCF) and eccentric-core fiber (ECF) [3, 4], long-period fiber gratings (LPFGs) [5-7] and tilted FBGs [8,9], they can operate in a large curvature range, but exhibit a lower sensitivity. For in-line interferometer-based curvature sensors, Mach-Zehnder interferometers (MZI) as a common and effective scheme usually involves two fiber mode-coupling joints, where the two joints act as a beam splitter and a beam combiner. For instance, the MZIs consisting of up-tapers, lateral-offsets, peanut-shape or spherical-shape structures [10-13] are proposed to sense the curvature variation, while they cannot distinguish the direction of the curvature due to the symmetrical structure. For achieving directional curvature sensitivity, an asymmetric index perturbation in the cross section of fiber by use of $\mathrm{CO}_{2}$ laser is introduced in the MZI, such as asymmetrical LPFG [14] and two tilted LPFGs [15], but their curvature sensitivity still need to be improved. In the meantime, the temperature cross-talk in the curvature measurement is another issue. In general, the strategy for avoiding the cross-talk is to simultaneously measure curvature and temperature by examining different interference peaks $[10,16]$ which possess different curvature and temperature responses. Another effective approach is to use special fibers in the MZIs for different temperature and curvature sensing mechanism, such as single-hole twin ECF [17], two-core fibers (TCF) [18], however, the fabrication and cost of these special fibers and control of splicing loss with standard single-mode fiber (SMF) need to be took into account.

In the work reported here, a small segment of D-shaped fiber is fusion spliced into a standard SMF, forming a MZI with $\mathrm{D}$-shaped fiber. When the D-shaped MZI is bent, the variation of the intermodal phase difference results in the shift of interference fringes with curvature change. The device can greatly enhance the curvature sensitivity and distinguish the orientation of curvature experienced by the fiber as the attenuation dip producing either a blue or red wavelength shift, by virtue of the asymmetry of D-shaped fiber cladding. Furthermore, a short FBG is inscribed in the D-shaped fiber, the temperature and curvature can be discriminately monitored in terms of their different response mechanisms and coefficients. Therefore, the proposed novel sensor based on a fiber MZI with embedded D-shaped FBG can be expected to achieve high-sensitivity directional curvature and temperature 
measurement simultaneously.

\section{DEVICE FABRICATION AND SENSING PRINCIPLE}

The schematic configuration of the proposed sensor is sketched in Fig. 1(a), which has a segment of $15 \mathrm{~mm}$ long D-shaped fiber spliced between SMFs with a small core-offset by a polarization-maintaining (PM) fiber arc fusion splicer (FSM-45PM). In the splicing process, the orientation of flat surface of $\mathrm{D}$-shaped fiber is rotated by $\theta$-axis motor of the splicer. The offset, arc power, arc time and other splicing parameters are precisely controlled by the fusion splicer program, ensuring the repeatable device fabrication. Light from a broadband source is coupled to the core mode and the asymmetric cladding modes of the D-shaped fiber at the first core-offset joint and then mixed at the second joint and partly coupled back to the core of lead-out SMF. The in-line D-shaped fiber MZI is therefore formed in terms of the optical phase difference between the core mode and the excited cladding modes. In the splicing process, the transmission spectrum was monitored by an optical spectrum analyzer (OSA) to ensure the desired interference. Figure 1(b) shows the microscopic images of the offset junctions along the $x-z$ and $y-z$ planes observed with the fusion splicer's camera, respectively, and the cross-section of D-shaped fiber. The radiuses of the core and circular cladding are $9.43 \mu \mathrm{m}$ and $62.5 \mu \mathrm{m}$, respectively, and the distance between the center of the core and the flat surface of the cladding is $\sim 7.89 \mu \mathrm{m}$. The photosensitivity of the MZI structure was firstly enhanced by $\mathrm{H}_{2}$ loading for 72 hours. For FBG inscription, we rotated the D-shaped fiber so that its flat surface faces the phase mask and UV-laser, avoiding the asymmetric edge diffraction with an improvement on the inscription efficiency. Hence, a 5mm long FBG was inscribed into the D-shaped fiber by scanning a phase mask with a frequency-doubled $\mathrm{Ar}^{+}$laser (244nm).

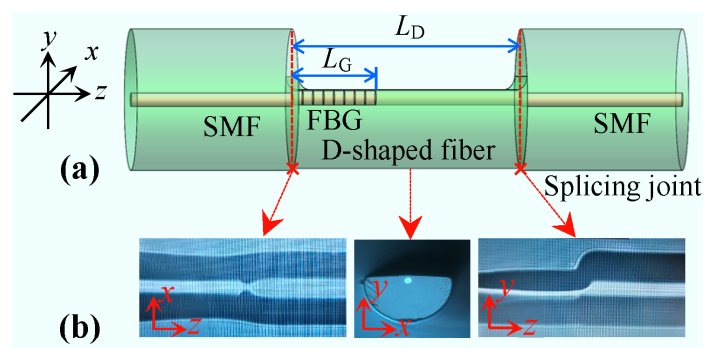

(c)

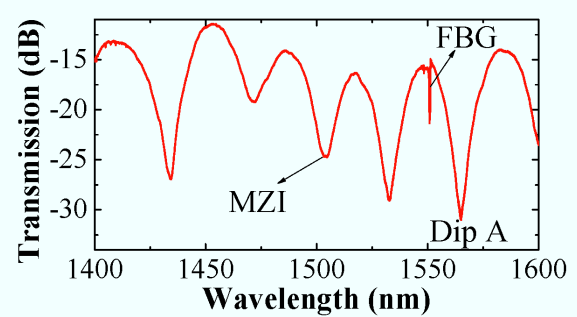

Fig. 1. (a) Schematic diagram of the MZI with embedded D-shaped FBG, (b) photographs of the splicing joints and D-shaped fiber section at different planes of $x-z, x-y$ and $y-z$, and (c) measured transmission spectrum.

Figure 1(c) shows the measured transmission spectrum of the MZI with D-shaped FBG. As expected, the interference fringes with a good visibility of $\sim 15 \mathrm{~dB}$ in the range of $1400 \sim 1600 \mathrm{~nm}$ are observed for the MZI, along with a Bragg resonant peak of the FBG around $1548 \mathrm{~nm}$.

As depicted in Fig. 1(a), in the MZI with D-shaped FBG structure, the two lateral core-offset joints act as the beam splitter and beam combiner. At the first core-offset joint, both cladding modes and core mode of the D-shaped fiber are excited due to mode field mismatching and then propagating in the asymmetry D-shaped fiber and passing through the FBG before mixing at the second joint. Light propagating in the cladding modes and core mode along the D-shaped fiber will accumulate a phase difference, giving rising to the interference at the output. In either case, the accumulated phase shift in each interference arm depends on the propagating length and the effective refractive index (RI) of the modes, and the phase difference $\Phi$ between the core and cladding modes can be approximated as [19-21]

$$
\Phi=\frac{2 \pi \Delta n_{\mathrm{eff}}(\lambda) L_{\mathrm{D}}}{\lambda}+\phi_{\mathrm{G}}(\lambda)
$$

where, $\Delta n_{\text {eff }}$ is the difference of the effective RI between the core and cladding modes of D-shaped fiber, $L_{\mathrm{D}}$ is the length of D-shaped fiber, and $\phi_{\mathrm{G}}$ is the additional phase difference induced by the FBG, which depends on the its RI modulation depth and grating length $L_{\mathrm{G}}$. Contribution of $\phi_{\mathrm{G}}$ in (1) depends on the wavelength and is only significant when $\lambda$ is close to $\lambda_{\mathrm{B}}$ because of strong contra-propagating coupling of the core mode. Therefore, with the phase difference satisfying the condition $\Phi=(2 m+1) \pi$ ( $m$ being the order of the MZI) for destructive interference, the resonant wavelength $\lambda_{\mathrm{m}}\left(\lambda_{\mathrm{m}} \neq \lambda_{\mathrm{B}}\right)$ of the attenuation dip can be found at

$$
\lambda_{m}=\frac{2 \Delta n_{\mathrm{eff}} L_{\mathrm{D}}}{2 m+1} .
$$

(a)

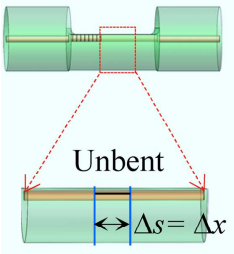

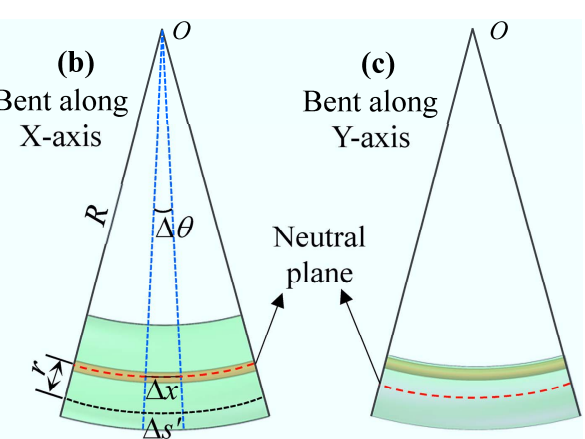

Fig. 2 Schematic of the bent beam based on a MZI with D-shaped FBG. (a) Unbent, (b) X-axis bent and (c) Y-axis bent D-shaped fiber section.

When the MZI is bent, the segment of D-shaped fiber is analogues to be deformed into a bend beam, and an incremental element is shown both unbent and bent beams in Fig. 2 . According to bent-beam theory of the material mechanics, there exists a neutral plane (or axis) for objects being bent and any line segment $\Delta x$ on the plane does not experience variation of length and thus strain. Line segment $\Delta s$ locating away from this neutral plane with a distance of $r$ will elongate or contract, becoming $\Delta s^{\prime}$ after deformation. Then by definition, the normal 
strain along $\Delta s$ is determined by

$$
\varepsilon=\lim _{\Delta s \rightarrow 0} \frac{\Delta s^{\prime}-\Delta s}{\Delta s} .
$$

Strain can be represented in terms of distance $r$ from the neutral axis and radius of curvature $R$ of the longitudinal axis of the element. Before deformation, $\Delta s=\Delta x$, but after deformation $\Delta x$ has a bending radius $R$ with the center point $O$. Since $\Delta \theta$ defines the angle between the cross sectional sides of the incremental element $\Delta s=\Delta x=R \Delta \theta$. Similarly, the deformed length of $\Delta s$ becomes $\Delta s^{\prime}=(R-r) \Delta \theta$. Substituting these relations into Eq. 3 gives the expression of the strain in the bent-beam as

$$
\varepsilon=\lim _{\Delta \theta \rightarrow 0} \frac{(R-r) \Delta \theta-R \Delta \theta}{R \Delta \theta}=-\frac{r}{R}=r \cdot C,
$$

where $C$ is the curvature of the bent $\mathrm{D}$-shaped fiber. Since the maximum strain occurs at the outer fiber cladding which is at the distance $r_{0}$ from the neural surface, $\varepsilon_{\max }=-r_{0} / R=\varepsilon_{0}$. Note the above results indicate that the longitudinal normal strain of any element within the fiber depends on its location $r$ on the cross section and the curvature of the fiber's longitudinal axis. In addition, a contraction will occur in the region above the neutral surface whereas elongation will arise in the region below the neutral surface. Therefore, for the bent D-shaped fiber, the effective RI difference induced by the strain difference $\Delta \varepsilon$ in the fiber core and cladding can be defined as $\Delta n_{\text {eff }}^{\mathrm{s}}=k \cdot \Delta \varepsilon=k \cdot d / R$, where $d$ is the distance between the fiber core and cladding, and $k$ is the strain-RI index coefficient. Hence, the wavelength shift of the attenuation dip can be given by

$$
\Delta \lambda_{m} \cong \frac{2 k L_{\mathrm{D}} d}{2 m+1} \cdot \Delta C
$$

From Eq. 5, it can be clearly seen that the wavelength of dip will linearly shift with the curvature of the bending fiber. Due to the asymmetric structure of D-shaped fiber, there will be different strain differences between core and cladding and then wavelength shift behaviors at two bending orientations. Thus, the MZI can be used directional bending sensing.

For the FBG inscribed in the D-shaped fiber, the resonant wavelength, given by $\lambda_{\mathrm{G}}=2 n_{\mathrm{eff}} \Lambda$, will also shift due to the bending, following [3]

$$
\Delta \lambda_{\mathrm{G}}=\left(1+P_{\mathrm{e}}\right) \lambda_{\mathrm{G}} r \cdot \Delta C,
$$

where $P_{\mathrm{e}}$ is the effective photo-elastic constant of the D-shaped fiber. Obviously, the wavelength shift is proportional to the change of the curvature and the FBG's position relative to the neutral axis, given by $r$. Therefore, the bending direction also affects $r$ and the wavelength shift manner of the FBG.

From Eq. 2 and FBG's resonant wavelength $\lambda_{\mathrm{G}}=2 n_{\text {eff }} \Lambda$, the influence of temperature on the dips of MZI's resonances and Bragg wavelength of the FBG can be expressed as

$$
\begin{aligned}
\Delta \lambda_{m}^{T} & =\frac{2}{2 m+1}\left(\Delta n_{\text {eff }} \frac{\partial L_{\mathrm{D}}}{\partial T} \Delta T+2 L_{\mathrm{D}} \frac{\partial\left(\Delta n_{\text {eff }}\right)}{\partial T}\right) \Delta T \\
& =\lambda_{m}\left(\alpha+\xi^{\prime}\right) \cdot \Delta T, \\
\Delta \lambda_{\mathrm{G}}^{T} & =2 n_{\text {eff }} \frac{\partial \Lambda}{\partial T} \Delta T+2 \Lambda \frac{\partial n_{\text {eff }}}{\partial T} \Delta T=\lambda_{\mathrm{G}}(\alpha+\xi) \cdot \Delta T,
\end{aligned}
$$

where, $\Delta T$ is the temperature change, $\alpha$ and $\xi$ are the thermo-expansion and thermo-optic coefficients of the D-shaped fiber, respectively, and $\xi^{\prime}$ is the variation coefficient of the effective RI difference between the core and cladding modes. It can be seen that the two wavelengths shift linearly with the change of temperature.

When the bend and temperature are applied to the sensing element, MZI responses with different coefficients with regard to FBG for each parameter, allowing to figure out the changes of curvature $\Delta C$ and temperature $\Delta T$ by using a coefficient matrix as [22]

$$
\left[\begin{array}{c}
\Delta C \\
\Delta T
\end{array}\right]=\frac{1}{D}\left[\begin{array}{cc}
K_{T, \mathrm{FBG}} & -K_{T, \mathrm{MZI}} \\
-K_{C, \mathrm{FBG}} & K_{C, \mathrm{MZI}}
\end{array}\right]\left[\begin{array}{c}
\Delta \lambda_{\mathrm{MZI}} \\
\Delta \lambda_{\mathrm{FBG}}
\end{array}\right],
$$

where, the determinant of the coefficient matrix $D=K_{C, \mathrm{MZI}} K_{T, \mathrm{FBG}}-K_{T, \mathrm{MZI}} K_{C, \mathrm{FBG}}$, the matrix coefficients $K_{C, \mathrm{MZI}}$, $K_{T, \mathrm{MZI}}, K_{C, \mathrm{FBG}}$, and $K_{T, \mathrm{FBG}}$ refer to the sensitivities of the MZI and FBG to the curvature and temperature, respectively. And also, as mentioned above, bending responses of both MZI and FBG are direction-sensitive, and $K_{C, \mathrm{FBG}}$ and $K_{C, \mathrm{MZI}}$ have different values for $\mathrm{X}$-axis and $\mathrm{Y}$-axis bending.

\section{EXPERIMENTAL RESULTS AND DISCUSSION}

Figure 3 depicts the experimental setup for the curvature measurement. Light from a broadband supercontinuum (superK) source is launched into the sensing device, and transmission is monitored by the OSA. For the measurement of curvature, one end of the device was clamped, and the other end was left free, avoiding unintended strain. A plastic capillary attached at the down side of flexible thin-metal strip is used as a supporting housing for the sensing element, allowing it straight and free from strain. Curvature imposed to the MZI, accompanying the bending of the thin-metal strip is controlled by a micrometer. The orientation of D-fiber in the MZI can be controlled with two fiber rotators. The length $2 L$ of the simply-supported beam for bending is $11 \mathrm{~cm}$, and the curvature of the sensing device can be calculated using $C=1 / R=2 y /\left(y^{2}+L^{2}\right)$, where $y$ is the displacement of the center of the fiber beam.

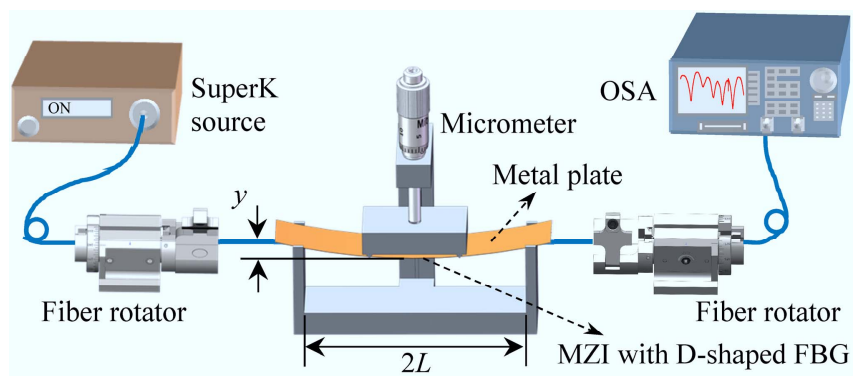

Fig. 3. Experimental setup for curvature measurement.

The evolution of transmission spectra for bending along $\mathrm{X}$-axis and $\mathrm{Y}$-axis are presented in Fig. 4(a) and dip A (marked in Fig. 1(c)) was monitored for measurement for its relatively deeper dip. The experimental results show that the dip A undergoes opposite shift (red or blue) for bending along the $\mathrm{X}$-axis and Y-axis, respectively. The shifts of dip A verses 
curvature for the two orthogonal directions are plotted in Fig. 4(b). During the bending process, the one end of the sensing element kept free after the orientation adjustment of D-shaped fiber, then the pure bend was applied to the fiber. In the curvature range of $0 \sim 0.3 \mathrm{~m}^{-1}$, the wavelength of dip A shifts by $24.25 \mathrm{~nm}$ and $-14.54 \mathrm{~nm}$ for $\mathrm{X}$-axis and $\mathrm{Y}$-axis bending, and the corresponding curvature sensitivities from the linear fitting results are $87.7 \mathrm{~nm} / \mathrm{m}^{-1}$ and $-48.9 \mathrm{~nm} / \mathrm{m}^{-1}$, respectively. The difference in curvature sensitivities along two orthogonal directions is simply due to the asymmetric cross-section of D-shaped fiber. As depicted in Fig. 2, when the D-shaped fiber is bent along $\mathrm{X}$-axis, the fiber cladding is much farther away from the neutral plane than that along $\mathrm{Y}$-axis, and then the former side of the fiber is elongated whereas the latter side is contracted. Therefore, a much larger stain difference between the fiber core and cladding along $\mathrm{X}$-axis bending leads to a much higher curvature sensitivity than that along $\mathrm{Y}$-axis bending. Furthermore, the existence of asymmetrical sections, i.e. the D-shaped fiber and two core-offset joints, played a dominated role in the high curvature sensitivity.
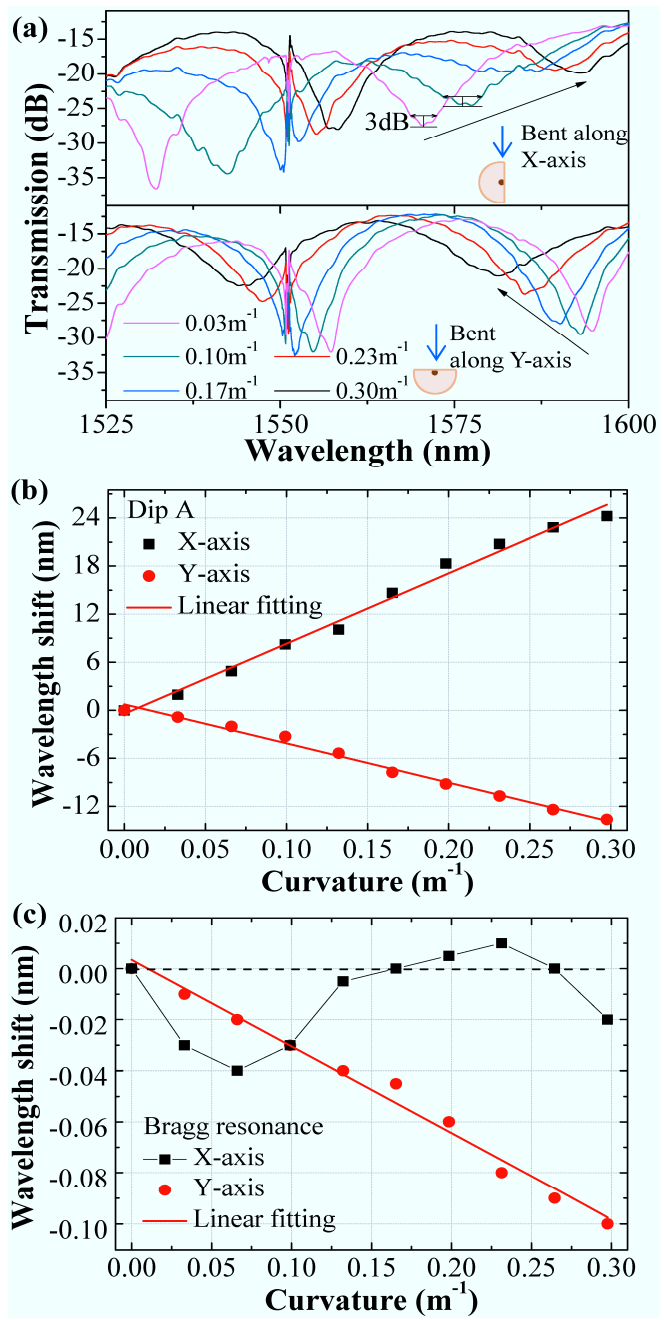

Fig. 4. When the MZI with D-shaped FBG is bent along two orthogonal directions, (a) evolution of transmission spectra, and the curvature responses of (b) dip A and (c) Bragg resonance.

Because the FBG was inscribed in the D-shaped fiber, the
Bragg wavelength also behaves differently for bending along two directions. Figure 4(c) shows a $0.1 \mathrm{~nm}$ blue-shift of the Bragg resonance (corresponding to the sensitivity of -0.33 $\mathrm{nm} / \mathrm{m}^{-1}$ ) when the bending happens along $\mathrm{Y}$-axis direction, causing a compressive strain of the fiber core. Whereas for $\mathrm{X}$-axis bending, as the FBG is almost located in the neutral plane of the fiber bent-beam, it only shows a fluctuation of maximum $0.04 \mathrm{~nm}$ (twice the resolution $0.02 \mathrm{~nm}$ of the OSA), which is mainly caused by a small positional deviation of fiber core with respect to the neutral plane and the inaccurate wavelength reading when the Bragg resonance is close to the resonant wavelength of MZI.

The temperature response of the MZI with D-shaped FBG was also examined by placing it in a heating chamber with a temperature controller. Figure 5(a) shows the spectral evolution with the increase of temperature, and it can be seen that both of dip A and Bragg resonance shift to longer wavelength. During the temperature measurement, since the Bragg wavelength of the FBG is close to the resonant wavelength of the MZI attenuation dip, the additional phase difference induced by the strong contra-propagating core-mode coupling of the FBG breaks the inherent interference condition. As a result, the transmission dip of FBG is converted to the peak. From the detailed measurement results in Fig. 5(b), the temperature sensitivities for dip A and Bragg resonance are calculated as $0.086 \mathrm{~nm} /{ }^{\circ} \mathrm{C}$ and $0.012 \mathrm{~nm} /{ }^{\circ} \mathrm{C}$, respectively.
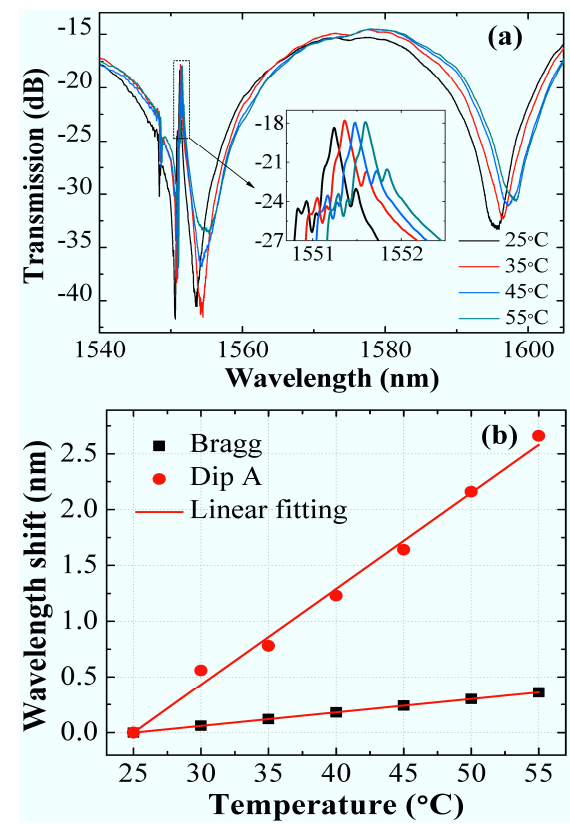

Fig. 5. (a) Spectral evolution with the temperature, and (b) the temperature responses of dip A and Bragg resonance of the MZI with D-shaped FBG.

Based on the above experimental results and Eq. 8, when the MZI with D-shaped FBG is bent along X- (or Y-) axis, the coefficient matrix of the curvature and temperature measurement can be constructed by

$$
\left[\begin{array}{c}
\Delta C \\
\Delta T
\end{array}\right]=\frac{1}{1.0524(-0.5584)}\left[\begin{array}{cc}
0.012 & -0.086 \\
0(0.33) & 87.7(-48.9)
\end{array}\right]\left[\begin{array}{l}
\Delta \lambda_{\mathrm{MZI}} \\
\Delta \lambda_{\mathrm{FBG}}
\end{array}\right],
$$

where the value in parentheses is for Y-axis bending. Therefore, 
the variations of the temperature and curvature can be monitored discriminately and simultaneously by using the coefficient matrix. And the bending orientation can be distinguished according to the blue or red shift of the attenuation dip. The maximum curvature sensitivity of $87.7 \mathrm{~nm} / \mathrm{m}^{-1}$ is much higher than that of most reported sensors. Table 1 gives the sensing performance in comparison with several previous reports based on MZI or FBG. It can be seen that the proposed sensor not only has high curvature sensitivity, but also achieve the simultaneous measurement of curvature and temperature, with the added advantage of direction recognition ability due to the asymmetric structure of D-shaped fiber.

TABLE 1. COMPARISON OF SENSING PERFORMANCE BETWEEN DIFFERENT MZI AND FBG-BASED CURVATURE SENSORS

\begin{tabular}{ccccc}
\hline Sensing scheme & $\begin{array}{c}\text { Cur. sensitivity } \\
\left(\mathrm{nm} / \mathrm{m}^{-1}\right)\end{array}$ & Direction & $\begin{array}{c}\text { Tem. sensitivity } \\
\left(\mathrm{pm} /{ }^{\circ} \mathrm{C}\right)\end{array}$ & Ref. \\
\hline $\begin{array}{c}\text { MZI with two } \\
\text { peanut-shape } \\
\text { structures }\end{array}$ & -21.87 & Insensitive & $\mathrm{N} / \mathrm{A}$ & {$[12]$} \\
\hline $\begin{array}{c}\text { MZI with core-offset } \\
\text { and spherical-shape }\end{array}$ & -22.227 & Insensitive & 84.7 & {$[10]$} \\
\hline $\begin{array}{c}\text { MZI with } \\
\text { lateral-offset and } \\
\text { up-taper }\end{array}$ & 11.987 & Insensitive & 68 & {$[16]$} \\
\hline $\begin{array}{c}\text { MZI with PCF } \\
\text { MZI with two tilted } \\
\text { LPFGs }\end{array}$ & -15.4935 & Sensitive & 40.6 & {$[15]$} \\
\hline $\begin{array}{c}\text { FBG written in ECF } \\
\text { and SMF }\end{array}$ & 0.05 & Sensitive & N/A & {$[3]$} \\
\hline $\begin{array}{c}\text { MZI with D-shaped } \\
\text { FBG }\end{array}$ & $\mathbf{8 7 . 7}$ & Sensitive & $\mathbf{8 6 . 0}$ & $\begin{array}{c}\text { This } \\
\text { work }\end{array}$ \\
\hline
\end{tabular}

\section{CONCLUSIONS}

We have proposed and demonstrated the use of an in-line fiber MZI with D-shaped FBG for directional curvature and temperature measurement simultaneously. The experimental results show that the proposed sensing structure has different curvature responses with respect to the bending direction, and the curvature sensitivities of MZI for $\mathrm{X}$-axis and $\mathrm{Y}$-axis bending are $87.7 \mathrm{~nm} / \mathrm{m}^{-1}$ and $-48.9 \mathrm{~nm} / \mathrm{m}^{-1}$ in the curvature range of $0 \sim 0.3 \mathrm{~m}^{-1}$, respectively. Depending on the embedded D-shaped FBG's location with respect to the neutral plane, the bending along the two orthogonal directions gives either almost insensitivity or a $0.1 \mathrm{~nm}$ blue-shift of Bragg wavelength. Moreover, the resonant wavelengths of the MZI and D-shaped FBG linearly change with the temperature, and the response coefficients are $0.086 \mathrm{~nm} /{ }^{\circ} \mathrm{C}$ and $0.012 \mathrm{~nm} /{ }^{\circ} \mathrm{C}$, respectively. Therefore, the proposed sensing structure exhibits decisive advantages of high-sensitive directional curvature measurement and simultaneous temperature measurement, presenting great potentials in practical applications such as structural health monitoring, industrial robots, and so on.

\section{REFERENCES}

[1] Y. Zhang, W. Zhang, Y. Zhang, S. Wang, L. Yu, and Y. Yan, "Simultaneous measurement of curvature and temperature based on LP
11 mode Bragg grating in seven-core fiber," Meas. Sci. Technol., vol. 28, no. 5, pp. 055101, Mar. 2017.

[2] A. Rauf, J. Zhao, B. Jiang, Y. Jiang, and W. Jiang, "Bend measurement using an etched fiber incorporating a fiber Bragg grating," Opt. Lett., vol. 38, no. 2, pp. 214-216, Jan. 2013.

[3] J. Kong, X. Ouyang, A. Zhou, H. Yu, and L. Yuan, "Pure directional bending measurement with a fiber Bragg grating at the connection joint of eccentric-core and single-mode fibers," J. Lightwave Technol., vol. 34, no. 14, pp. 3288-3292, Jul. 2016.

[4] G. M. H. Flockhart, W. N. MacPherson, J. S. Barton, J. D. C. Jones, L. Zhang, and I. Bennion, "Two-axis bend measurement with Bragg gratings in multicore optical fiber," Opt. Lett., vol. 28, no. 6, pp. 387-389, Mar. 2003.

[5] P. Saffari, T. Allsop, A. Adebayo, D. Webb, R. Haynes, and M. M. Roth, "Long period grating in multicore optical fiber: an ultra-sensitive vector bending sensor for low curvatures," Opt. Lett., vol. 39, no. 12, pp. 3508-3511, Jun. 2014.

[6] P. Geng, W. Zhang, S. Gao, H. Zhang, J. Li, S. Zhang, Z. Bai, and L. Wang, "Two-dimensional bending vector sensing based on spatial cascaded orthogonal long period fiber," Opt. Express, vol. 20, no. 27, pp. 28557-28562, Dec. 2012

[7] Q. Zhou, W. Zhang, L. Chen, Z. Bai, L. Zhang, L. Wang, B. Wang, and T. Yan, "Bending vector sensor based on a sector-shaped long-period grating," IEEE Photon. Technol. Lett., vol. 27, no. 7, pp. 713-716, Apr. 2015.

[8] L.-Y. Shao, L. Xiong, C. Chen, A. Laronche, and J. Albert, "Directional bend sensor based on re-grown tilted fiber Bragg grating," J. Lightwave Technol., vol. 28, no. 18, pp. 2681-2687, Sep. 2010.

[9] D. Feng, W. Zhou, X. Qiao, and J. Albert, "Compact optical fiber 3D shape sensor based on a pair of orthogonal tilted fiber Bragg gratings," Sci. Rep. vol. 5, pp. 17415, Nov. 2015.

[10] H. Gong, M. Xiong, Z. Qian, C. L. Zhao, and X. Dong, "Simultaneous measurement of curvature and temperature based on Mach-Zehnder interferometer comprising core-offset and spherical-shape structures," IEEE Photon. J., vol. 8, no. 1, pp. 1-9, Feb. 2016.

[11] C. Zhang, J. Zhao, C. Miao, H. Li, H. Bai, and M. Zhang, "Curvature and temperature sensor based on bulge-taper structures interferometer with embedded fiber Bragg grating," Opt. Eng., vol. 54, no. 8, pp. 087104-087104, Aug. 2015.

[12] H. Gong, X. Yang, K. Ni, C. L. Zhao, and X. Dong, "An optical fiber curvature sensor based on two peanut-shape structures modal interferometer," IEEE Photon. Technol. Lett., vol. 26, no. 1, pp. 22-24, Jan. 2014.

[13] B. Sun, Y. Huang, S. Liu, C. Wang, J. He, C. Liao, G. Yin, J. Zhao, Y. Liu, J. Tang, J. Zhou, and Y. Wang, "Asymmetrical in-fiber Mach-Zehnder interferometer for curvature measurement," Opt. Express, vol. 23, no. 11, pp. 14596-14602, Jun. 2015.

[14] W. Ni, P. Lu, C. Luo, X. Fu, L. Liu, H. Liao, X. Jiang, D. Liu, and J. Zhang, "Bending direction detective fiber sensor for dual-parameter sensing based on an asymmetrical thin-core long-period fiber grating," IEEE Photon. J., vol. 8, no. 4, pp. 1-11, Aug. 2016.

[15] Y. P. Li, W. G. Zhang, S. Wang, L. Chen, Y. X. Zhang, B. Wang, T. Y. Yan, X. Y. Li, and W. Hu, "Bending vector sensor based on a pair of opposite tilted long-period fiber gratings," IEEE Photon. Technol. Lett., vol. 29, no. 2, pp. 224-227, Jan. 2017.

[16] S. Zhang, W. Zhang, S. Gao, P. Geng, and X. Xue, "Fiber-optic bending vector sensor based on Mach-Zehnder interferometer exploiting lateral-offset and up-taper," Opt. Lett., vol. 37, no. 21, pp. 4480-4482, Nov. 2012.

[17] W. Ni, P. Lu, J. Zhang, C. Yang, X. Fu, Y. Sun, H. Liao, and D. Liu, "Single hole twin eccentric core fiber sensor based on anti-resonant effect combined with inline Mach-Zehnder interferometer," Opt. Express, vol. 25, no. 11, pp. 12372-12380, May 2017.

[18] O. Frazao, S. F. O. Silva, J. Viegas, J. M. Baptista, J. L. Santos, J. Kobelke, and K. Schuster, "All fiber Mach-Zehnder interferometer based on suspended twin-core fiber," IEEE Photon. Technol. Lett., vol. 22, no. 17, pp. 1300-1302, Sep. 2010.

[19] N. L. P. Andrews, R. Ross, D. Munzke, C. van Hoorn, A. Brzezinski, J. A. Barnes, O. Reich, and H.-P. Loock, "In-fiber Mach-Zehnder interferometer for gas refractive index measurements based on a hollow-core photonic crystal fiber," Opt. Express, vol. 24, no. 13, pp. 14086-14099, Jun. 2016. 
[20] R. Jha, J. Villatoro, G. Badenes, and V. Pruneri, "Refractometry based on a photonic crystal fiber interferometer," Opt. Lett., vol. 34, no. 5, pp. 617-619, Mar. 2009.

[21] P. Lu, L. Men, K. Sooley, and Q. Chen, "Tapered fiber Mach-Zehnder interferometer for simultaneous measurement of refractive index and temperature," Appl. Phys. Lett., vol. 94, no. 13, pp. 131110, Apr. 2009.

[22] Z. Kang, X. Wen, C. Li, J. Sun, J. Wang, and S. Jian, "Up-taper-based Mach-Zehnder interferometer for temperature and strain simultaneous measurement," Appl. Opt., vol. 53, no. 12, pp. 2691-2695, Apr. 2014. 